\title{
Simulation of MILD combustion using Perfectly Stirred Reactor model
}

(Accepted for publication in Proc. Combust. Inst., 01/06/2016)

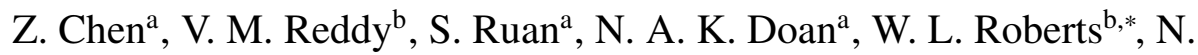 \\ Swaminathan $^{\mathrm{a}}$ \\ ${ }^{a}$ Department of Engineering, University of Cambridge, Trumpington Street, Cambridge, CB2 \\ 1PZ, United Kingdom \\ ${ }^{b}$ Clean Combustion Research Center, King Abdullah University of Science and Technology, \\ Thuwal, Saudi Arabia
}

\begin{abstract}
A simple model based on a Perfectly Stirred Reactor (PSR) is proposed for moderate or intense low-oxygen dilution (MILD) combustion. The PSR calculation is performed covering the entire flammability range and the tabulated chemistry approach is used with a presumed joint probability density function (PDF). The jet, in hot and diluted coflow experimental set-up under MILD conditions, is simulated using this reactor model for two oxygen dilution levels. The computed results for mean temperature, major and minor species mass fractions are compared with the experimental data and simulation results obtained recently using a multi-environment transported PDF approach. Overall, a good agreement is observed at three different axial locations for these comparisons despite the overpredicted peak value of $\mathrm{CO}$ formation. This suggests that MILD combustion can be effectively modelled by the proposed PSR model with lower computational
\end{abstract}

\footnotetext{
${ }^{*}$ Corresponding author.

Email address: william.roberts@kaust.edu.sa (W. L. Roberts)
} 
cost.

Keywords: Moderate or Intense Low-oxygen Dilution (MILD); Flameless

Combustion; Perfectly Stirred Reactor (PSR); Jet in Hot Coflow (JHC);

Correlated joint PDF

\section{Introduction}

Enhancement of thermal efficiency with ultralow emissions is one of the most challenging subjects for combustion researchers. Among the many techniques developed for this challenge, moderate or intense low-oxygen dilution (MILD) combustion has been identified as a promising concept to achieve these goals. MILD combustion has been well defined in many previous studies [1-7], and it is characterized by a reactant temperature, $T_{\mathrm{r}}$, higher than the reactant mixtures autoignition temperature $T_{\mathrm{ign}}$, and the temperature rise in the combustor, $\Delta T=$ $T_{\mathrm{p}}-T_{\mathrm{r}}$, is smaller than $T_{\mathrm{ign}}$. The efficiency increases due to heat recirculation from the combustion products, and with reduced emissions since the maximum temperature is decreased as a result of dilution and more homogeneous reaction in the combustor [8-13].

Since MILD combustion is an attractive novel technique, it is intensively studied experimentally and numerically to understand its principal mechanisms and develop appropriate models to help design combustors under MILD conditions. In particular, the Adelaide Jet in Hot Coflow (JHC) burner of Dally et al. [14] has received much attention from the modelling community due to the comprehensive set of measured data available. Christo and Dally [15] compared measurements with simulation results using conserved scalar-based (e.g. flamelet approaches) and Eddy-Dissipation Concept (EDC) models. The scalar-based models were 
found to be inadequate to model MILD combustion, whereas the EDC model was able to reasonably capture the radial distributions for major species. Aminian et al. [16] further explored the capabilities of EDC models by modifying the chemical time constant, and improved results were obtained suggesting that the lower gradient of species and temperature in MILD combustion leads to a longer residence time in the fine structure of turbulence than in conventional premixed combustion. Ihme et al. [17] modelled the burner as a three-stream problem using Large Eddy Simulation (LES) with a flamelet/progress variable (FPV) formulation. An additional conserved scalar was introduced to account for the effect of the entraining air in the downstream. Conditional Moment Closure (CMC) was also applied [18] using a single mixture fraction and a detailed 47-species chemical mechanism. Lee et al. [19] recently used a Multi-environment Probability Density Function (MPDF) approach for the Adelaide JHC configuration. Excellent overall agreement was obtained in these modelling works despite the relatively high computational cost.

A recent study by De and Dongre [20] extensively compared various models used for MILD combustion including the steady flamelet model, transported PDF models (Lagrangian (LPDF) and Multi-environment approaches) as well as EDC based models. The results showed that only the LPDF model resulted in a reasonable agreement with experimental data for major species and mean temperatures, whereas substantial discrepancies were still found for minor species such as $\mathrm{OH}$ and $\mathrm{CO}$. These numerical studies have indeed improved the physical understanding of MILD combustion. However, the models based on conventional flames such as flamelet and EDC approaches seem inadequate as they assume that reactions occur in either smaller or similar scales compared to smallest turbulence 
structures. The EDC model assumes that the reactor is of the Kolmogorov scale size, which is acceptable for large Damköhler number, $\mathrm{Da}>>1$. On the contrary, it was observed in experiments [21,22] that reactions take place in relatively large structures under MILD conditions and Da is likely to be of order 1 or below. The sophisticated transported PDF and CMC methods are too expensive computationally for practical geometries. Thus, there is a need for a simple but adequate model to be proposed for MILD combustion.

The spatially distributed MILD reaction zones were shown [6] to have nonnegligible tangential gradient of temperature and species compared to its normal gradient. Based on this physical insight, the DNS analysis by Minamoto and Swaminathan $[23,24]$ suggested that the Perfectly Stirred Reactor (PSR) may be used as a suitable canonical candidate for MILD combustion modelling. This approach is different from EDC because it does not presume $\mathrm{Da}>>1$, and the local state of the PSR is random and its mean state is determined statistically (see Section 2) as opposed to the deterministic approach used in the EDC. To further explore this concept, the objective of this paper is to propose a modelling framework based on the PSR. This framework was originally developed for partially premixed flames $[25,26]$ and here it is modified for MILD combustion. Details about this reactor model are elaborated in Section 2. The model is then applied to the Adelaide JHC burner [14] as described in Section 3 and results are compared with the measured data and computational results obtained using a Multi-environment PDF method [19] in Section 4. Conclusions are summarised in Section 5. 


\section{Mathematical modelling}

\subsection{Governing equations}

To simulate this JHC configuration, the Favre-averaged equations for mass, momentum and total enthalpy are solved. The standard two-equation $\widetilde{k}-\widetilde{\epsilon}$ model is used for the turbulent jet flow. The model constant $C_{\epsilon 1}$ is modified from the standard value of 1.44 to 1.6 following the earlier studies $[15,19,27]$ to capture the spreading in a round jet. For this three-stream JHC configuration, two mixture indicators are required to describe the scalar mixing between the streams. Bilger's mixture fraction [28], $Z$, is used to represent the turbulent mixing between the fuel jet and the hot co-flow. Another conserved scalar, $F$, representing the inert mixing between the air entrainment and the hot co-flow is used and henceforth referred to as the mixing factor in this paper. The mixing factor $F=1$ denotes the air entrainment stream and $F=0$ implies the hot co-flow stream. Note that the influence of air entrainment on the reaction is not considered here since this only occurs in the far downstream as reported in [14]. For chemical reactions, a progress variable, $c$, is employed and its definition is further discussed in Section 2.2. The Favre transport equations of the first two moments for the mixture fraction, $\widetilde{Z}$ and $\widetilde{Z^{\prime \prime 2}}$, and progress variable, $\widetilde{c}$ and $\widetilde{c^{\prime \prime}}$, are solved in the simulations. The covariance, $\widetilde{Z^{\prime \prime} c^{\prime \prime}}$, signifying the statistical correlation between $Z$ and $c$ is also considered. These transport equations have been detailed in the modelling framework of $[25,26]$ and here in this paper we emphasise the closure models for MILD combustion in the $\widetilde{c}$ and $\widetilde{c^{\prime \prime}}$ equations written as

$\bar{\rho} \widetilde{\mathcal{D}}_{t} \widetilde{c}=\nabla \cdot\left(\overline{\rho D \nabla c}-\overline{\rho \boldsymbol{u}^{\prime \prime} c^{\prime \prime}}\right)+\overline{\dot{\omega}_{c}}$ 


$$
\begin{aligned}
\bar{\rho} \widetilde{D}_{t} \widetilde{c^{\prime \prime 2}}= & \nabla \cdot\left(\overline{\rho D \nabla c^{\prime \prime 2}}-\overline{\rho \boldsymbol{u}^{\prime \prime} c^{\prime \prime 2}}\right)-2 \bar{\rho} \widetilde{\chi}_{c} \\
& -2 \overline{\rho \boldsymbol{u}^{\prime \prime} c^{\prime \prime}} \cdot \nabla \widetilde{c}+2 \overline{c^{\prime \prime} \dot{\omega}_{c}^{\prime \prime}}
\end{aligned}
$$

where $\widetilde{\mathcal{D}}_{t} \equiv\left(\partial_{t}+\widetilde{\boldsymbol{u}} \cdot \nabla\right)$ is the substantial derivative and $D$ is the molecular diffusivity. The gradient transport approximation is used to model the turbulent scalar fluxes, for instance, $\overline{\rho \boldsymbol{u}^{\prime \prime} c^{\prime \prime}}=-\bar{\rho} D_{t} \nabla \widetilde{c}$, where $D_{t}$ is the turbulent diffusivity computed as $D_{t}=v_{t} / \mathrm{Sc}_{t}$ and the turbulent Schmidt number $\mathrm{Sc}_{t}$ is taken as 0.7 in this study. For the scalar dissipation rate of progress variable, the algebraic model proposed in $[29,30]$ is modified here for MILD combustion and this model is written as

$\widetilde{\chi}_{c} \simeq\left(\left[2 K_{c}^{*}-\tau C_{4}\right] \frac{1}{t_{c}}+C_{3} \frac{1}{t_{\mathrm{f}}}\right) \frac{\widetilde{c^{\prime \prime 2}}}{\beta^{\prime}}$,

where $t_{c}=\left(\overline{\dot{\omega}_{c}} / \bar{\rho}\right)^{-1}$ is a local chemical time scale with $\bar{\rho}$ being the mixture density and the model for the mean reaction rate $\overline{\dot{\omega}_{c}}$ is discussed later in Section 2.2. The local flow time scale, $t_{\mathrm{f}}$, is given as $t_{\mathrm{f}}=\widetilde{\varepsilon} / \widetilde{k}$. The model parameters, $\beta^{\prime}=6.7$, $C_{3}=1.5 \sqrt{\mathrm{Ka}} /(\sqrt{\mathrm{Ka}}+1)$, and $C_{4}=1.1 /(1+\mathrm{Ka})^{0.4}$, are kept to be the same as in a number of previous studies [25, 26, 29-34]. The Karlovitz number is defined as $\mathrm{Ka}=t_{c} / \sqrt{v / \widetilde{\epsilon}}$, where $v$ is the local kinematic viscosity. $K_{c}^{*}=0.87 \tau$ [29] and $\tau=\tau(Z)$ is the heat release parameter, varying within the flammable mixture range depending on the local equivalence ratio. These values are obtained from the PSR calculations.

The reaction source terms in Eqs. (1) and (2), $\overline{\dot{\omega}_{c}}$ and $\overline{c^{\prime \prime} \dot{\omega}_{c}^{\prime \prime}}$, require closure models for MILD combustion which differ from the flame models proposed in [25, 26], and these models are described next. 

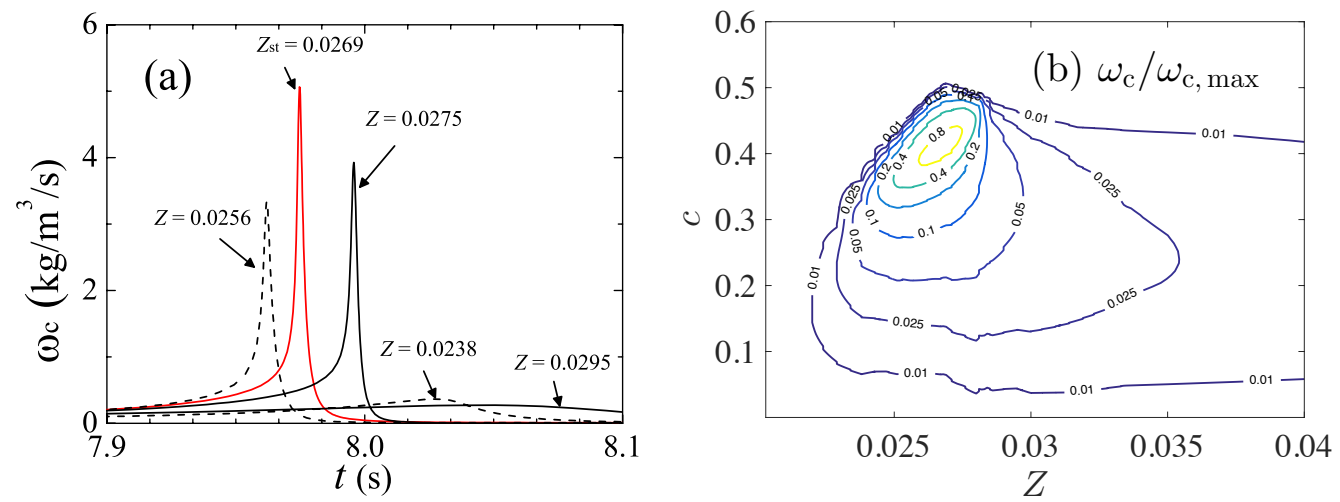

Fig. 1. Reaction rate of progress variable in the Perfectly Stirred Reactor. (a) Temporal variation for five typical mixture fractions. (b) Distribution in the mixture fraction and progress variable spaces.

\subsection{Perfectly Stirred Reactor model}

Zero-dimensional unsteady adiabatic PSR equations with two inflow streams are solved for different mixture fractions using CHEMKIN-PRO [35]. The composition and temperature conditions of fuel and hot oxidiser streams are kept the same as in the experiments [14]. The detailed chemical mechanism GRI-Mech 3.0 is used for the methane-hydrogen flames considered in this study. This mechanism has been used in the past for various numerical studies of MILD combustion [1719] and showed very good performance compared to other mechanisms [15]. For a particular mixture fraction, the progress variable is defined using temperature written as

$c(t)=\frac{T(t)-T_{\mathrm{f}}}{T_{\mathrm{b}}-T_{\mathrm{f}}}$,

where $T(t)$ is the reactor temperature at time $t, T_{\mathrm{f}}=305 \mathrm{~K}$ is the temperature of the fuel stream and $T_{\mathrm{b}}$ is the burnt equilibrium temperature. Thus, the reaction 
rate of the progress variable is given by

$\dot{\omega}_{c}(t)=\frac{\dot{q}(t)}{c_{p, \text { mix }}(t)\left[T_{\mathrm{b}}-T_{\mathrm{f}}\right]}$,

where $\dot{q}(t)$ is the volumetric heat release rate and $c_{p, \text { mix }}(t)$ is the specific heat capacity of the mixture. For convectional flame simulations, one could also use definition of $c$ based on major species mass fractions. However, this is not so for this JHC configuration because of the presence of major combustion products in the co-flow and two fuel species present in the jet fluid. Therefore, the progress variable based on temperature is chosen here for its simplicity. Based on the testing in $[23,24]$, a relatively long residence time is chosen in such a way that the entire range from reactor quenching to fully burning state is covered. Figure 1(a) shows a typical temporal variation of $\dot{\omega}_{c}$ in the PSR for a few typical mixtures for the $3 \%$ $\mathrm{O}_{2}$ case in the Adelaide JHC [14]. The high reaction rates occur around the stoichiometry and the most reactive mixture (earliest reaction) is slightly lean as one would expect. Moving away from the stoichiometry (red line), the reaction rate becomes quite small occurring at a later time for both the lean (dashed lines) and rich sides (solid lines). Using the definition of $c$ in Eq. (4), all the thermo-chemical quantities can be expressed as a function of $Z$ and $c$, for example, the mixture heat capacity $C p=C p(Z, c)$. The distribution of the normalised PSR reaction rate, $\dot{\omega}_{c}(Z, c) / \dot{\omega}_{c}(Z, c)_{\max }$, is presented in Fig.1(b) using contours. It is shown that the reaction rate is high in a small region near stoichiometry $(Z=0.027)$, where $c$ value is between 0.3 and 0.5 . This value of progress variable is relatively small compared to conventional premixed flames using unheated reactants because of the high temperature of the oxidiser. Although the rich flammability limit extends up to $Z=0.3$ due to the high oxidiser temperature, the reaction rate becomes quite small after $Z>0.03$. 
To model the mean reaction rate in the JHC, a presumed correlated joint PDF approach is used as in $[25,26]$ to account for the turbulent fluctuations. This model is written as

$$
\overline{\dot{\omega}}_{c}=\int_{0}^{1} \int_{0}^{1} \dot{\omega}_{c}(\xi, \zeta) P(\xi, \zeta) d \xi d \zeta,
$$

where $\xi$ and $\zeta$ are the sample space variables for the mixture fraction and progress variable respectively. The correlated joint $\operatorname{PDF} P(\xi, \zeta)$ is obtained using beta distributions and copula $[25,30]$. Similar closure is used to model the reaction term $\overline{c^{\prime \prime} \dot{\omega}_{c}^{\prime \prime}} \simeq \overline{c^{\prime \prime} \dot{\omega}_{c}}$ in Eq.(2).

Other Favre-averaged themo-chemical quantities such as mixture molecular weight and species mass fractions, denoted by vector $\boldsymbol{\Psi}$, are computed using the integral, $\boldsymbol{\Psi}=\int_{0}^{1} \int_{0}^{1} \boldsymbol{\Psi}(\xi, \zeta) P(\xi, \zeta) d \xi d \zeta$, where $\boldsymbol{\Psi}(\xi, \zeta)$ is the PSR value. In the simulation, the temperature, $\widetilde{T}$, is calculated using the definition of $c$ in Eq. (4) and hence $\widetilde{T}=\widetilde{c}\left(T_{\mathrm{b}}-T_{\mathrm{f}}\right)+T_{\mathrm{f}}$. The mean density is computed using the ideal gas state equation, $\bar{\rho}=\bar{p} W_{\text {mix }} / R_{0} \widetilde{T}$, with $R_{0}$ being the universal gas constant and $W_{\text {mix }}$ is mixture molecular weight. Detailed description of these procedures can be found in $[25,26,34]$.

It should be noted that the present model described above is a form of tabulated chemistry approach but not like that in [36]. As noted in the Introduction, gradients in the tangential directions of MILD reaction zones are not negligible and so PSR is used as a canonical reactor.

\section{Experiments and numerical setup}

The MILD combustion experiment conducted by Dally et al. [14] is selected as the test case for model validation. A schematic of the experimental setup is 
shown in Fig. 2. The central fuel jet with internal diameter of $4.25 \mathrm{~mm}$ is arranged in the annulus hot coflow of $82 \mathrm{~mm}$ diameter. The unheated fuel jet consists of $50 \%$ methane and $50 \%$ hydrogen based on mole fraction with the bulk mean jet velocity of $62 \mathrm{~m} / \mathrm{s}$ corresponding to a Reynolds number of approximately 10,000. The hot coflow mean velocity is $3.2 \mathrm{~m} / \mathrm{s}$ and three $\mathrm{O}_{2}$ levels of 3,6 , and $9 \%$ designated as HM1, HM2 and HM3, were considered in the experiments [14]. The burner was placed in a wind tunnel where the cold air entrainment velocity is the same as the coflow.

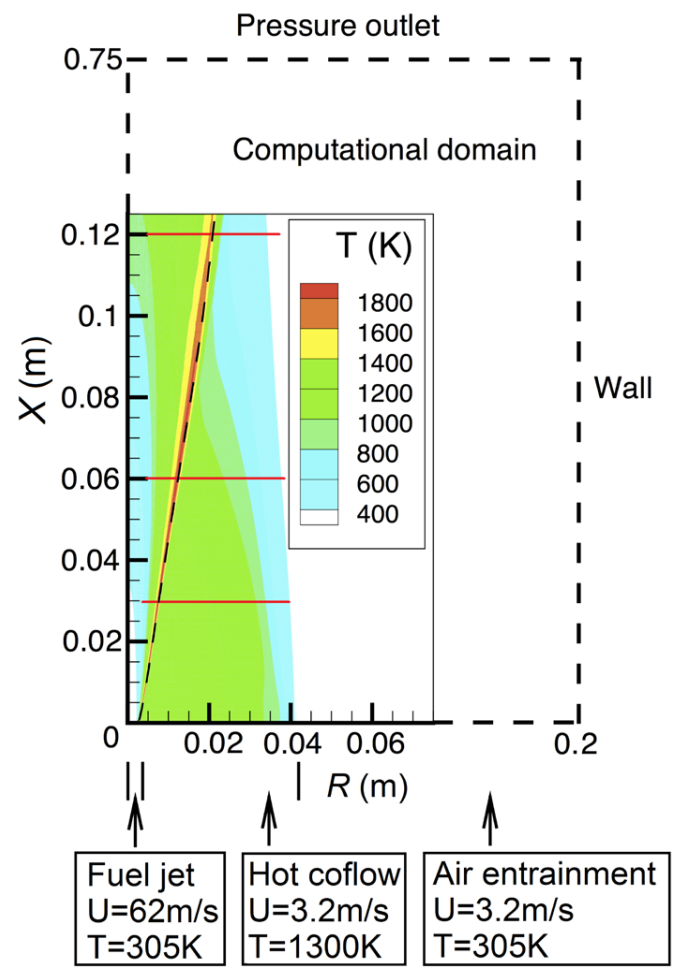

Fig. 2. Schematic of experimental geometry and computational domain. Temperature field for HM3 is shown for the region of interest and the horizontal lines denote measurement locations. The stoichiometric mixture fraction is shown as the dashed line.

In the present study, the two extreme cases of HM1 and HM3 are considered 
as detailed in Table 1. A simplified axisymmetric computational domain was used as shown in Fig. 2 along with the boundary conditions. The computed temperature field for HM3 is also presented for the region where the measurement are conducted (horizontal lines). The domain starts from the burner exit plane, and extends to $750 \mathrm{~mm}$ downstream in the axial direction $X$ and $200 \mathrm{~mm}$ in the radial direction. At the fuel inlet, a power law velocity profile is used and the turbulent intensity is set to 5\%. Top-hat velocity profiles are employed for both the hot coflow and air entrainment. For the scalars at the inlet boundaries, the mixture fraction is 1 in the fuel inlet and 0 in other streams. The progress variable is set to 1.0 for the fully burnt hot coflow and 0.0 for the cold jet and air inflows. A grid independence study was carried out using three different mesh sizes and the final grid with 141,000 cells was then chosen based on velocity and temperature comparisons at 30 and $120 \mathrm{~mm}$ axial locations.

Table 1. Hot coflow conditions $\left(Y_{i}\right.$ in $\left.\%\right)$.

\begin{tabular}{cccccc}
\hline Case & $\mathrm{T}(\mathrm{K})$ & $Y_{\mathrm{O}_{2}}$ & $Y_{\mathrm{N}_{2}}$ & $Y_{\mathrm{CO}_{2}}$ & $Y_{\mathrm{H}_{2} \mathrm{O}}$ \\
\hline $\mathrm{HM} 1$ & 1300 & 3 & 85 & 5.5 & 6.5 \\
$\mathrm{HM} 3$ & 1300 & 9 & 79 & 5.5 & 6.5 \\
\hline
\end{tabular}

The PSR combustion model noted in Section 2 is implemented in the commercial CFD code FLUENT 13.0 following the procedure described in [25]. The source terms and various thermo-chemical quantities are computed and tabulated prior to the simulations. The look-up table consists of five control parameters with the resolution of $80(\widetilde{Z}) \times 41(\widetilde{c}) \times 15\left(\widetilde{Z^{\prime \prime 2}}\right) \times 21\left(\widetilde{c^{\prime \prime 2}}\right) \times 11\left(\widetilde{Z^{\prime \prime} c^{\prime \prime}}\right)$, and it is generated using 80-core parallel computing which requires about 1 hour. Once the tabulation is complete, converged solution for the turbulent case can be obtained in about 30 
minutes using a 4-core desktop computer, which is several orders of magnitude faster than the transported PDF methods.

\section{Results and model comparison}

In this section, the computational results obtained using the PSR model are compared with experimental data from Dally et al. [14]. The results of Lee et al. [19] using a multi-environment PDF model are also used for comparison, and despite its high computational cost this particular study is chosen because it shows very good agreement with the experimental data compared to earlier studies using the $\operatorname{EDC}[15,16]$ and flamelet $[17,20]$ models.

Figure 3 shows the radial distribution of Favre mean temperature at three axial positions, $X=30,60$ and $120 \mathrm{~mm}$ for both flames HM1 (left column) and HM3 (right column). The values predicted by the PSR are in good agreement with the experimental measurements at all three axial locations for both flames. In the shear region between the fuel jet and hot coflow, combustion temperature increase of $100 \mathrm{~K}$ in $\mathrm{HM} 1$ and $500 \mathrm{~K}$ in $\mathrm{HM} 3$ are observed in the experiments, and are well captured by both the PSR and PDF models. This temperature rise occurs in a narrow region presenting a sharp tip as shown more visibly using the temperature contour in Fig. 2. However, the temperature is over-estimated in the outer mixing layer $(R>0.03 \mathrm{~m})$ between the cold air and hot coflow at most locations, which is possibly due to the simple interpolation applied using the mixing factor $F$. It is also noted that the PSR slightly over-predicts the peak temperature by about 2-3\% at $X=60$ and $120 \mathrm{~mm}$ for HM3. This may be due to the temperature approximation using Eq. (4) which neglects the turbulent fluctuation of $T_{\mathrm{b}}$. It is believed that these

discrepancies in temperature can be improved by solving a transport equation for 
enthalpy, and this will be explored in subsequent studies.

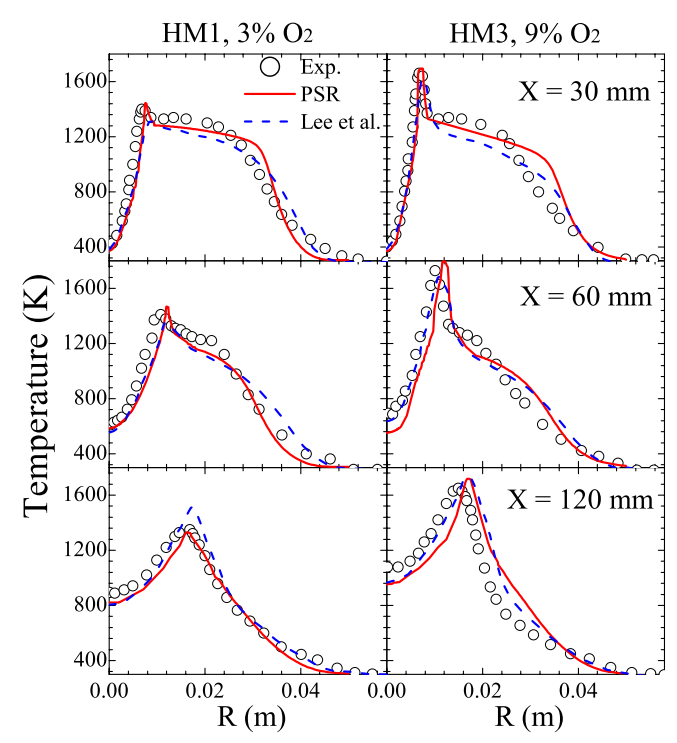

Fig. 3. Radial profiles of mean temperature.

The radial profiles of the mean mass fractions of the major reaction products, $\mathrm{CO}_{2}$ and $\mathrm{H}_{2} \mathrm{O}$, are shown in Figs. 4 and 5 respectively. The over-prediction of $\mathrm{CO}_{2}$ mass fraction with the PSR model in the outer inert mixing layer noted earlier is still seen, which improves as one moves downstream in Fig. 4. In contrast, the PDF model tends to capture the mixing between the shroud air and hot coflow quite well, but it over-estimates the peak values in the reaction zone for HM3 at all axial locations as well as $X=120 \mathrm{~mm}$ for HM1. The peak values obtained using the PSR model, however, are in excellent agreement with the experimental data although the predicted peak location at $X=120 \mathrm{~mm}$ is slightly shifted outwards radially for both flames. As for $\mathrm{H}_{2} \mathrm{O}$ mass fractions, an overall good agreement is seen in Fig. 5 between the measurements and both PSR and PDF models.

Combining the comparisons between the experimental data and computed 


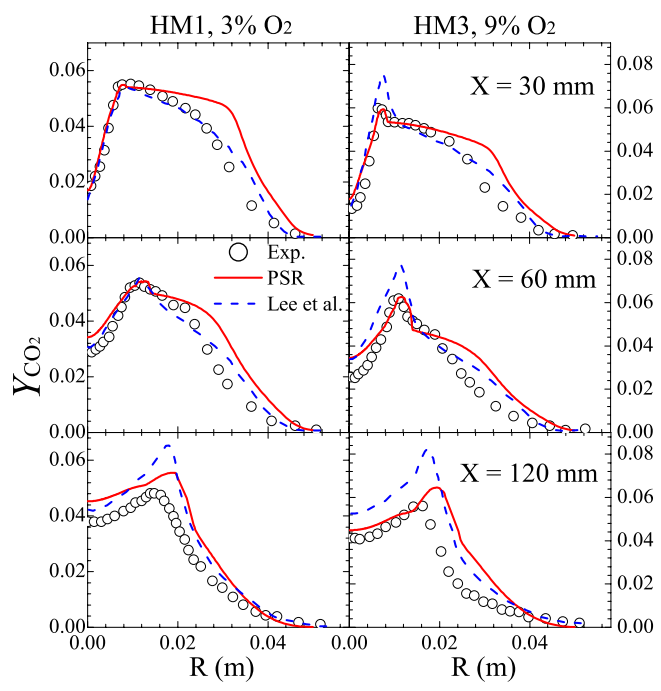

Fig. 4. Radial profiles of $\mathrm{CO}_{2}$ mass fraction.

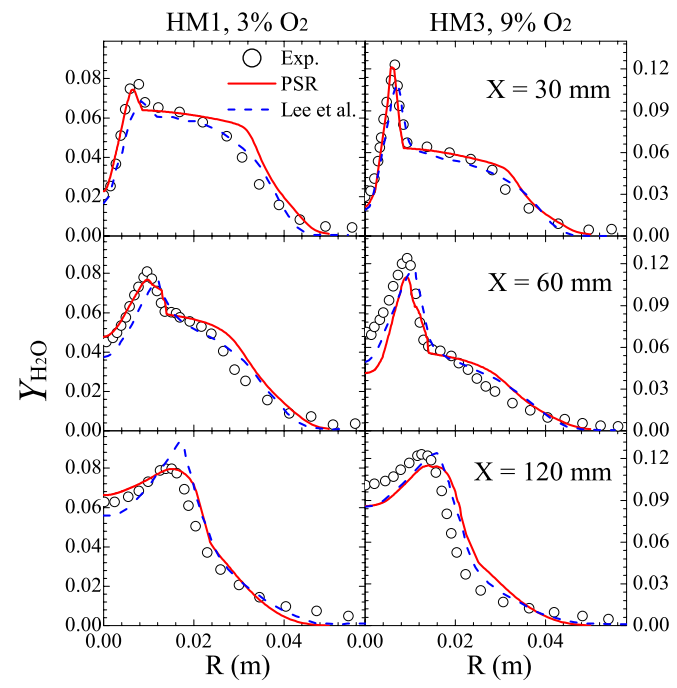

Fig. 5. Radial profiles of $\mathrm{H}_{2} \mathrm{O}$ mass fraction.

PSR results for temperature, and mass fractions of $\mathrm{CO}_{2}$ and $\mathrm{H}_{2} \mathrm{O}$, it can be seen that the agreement is generally better for flame HM1 with $3 \% \mathrm{O}_{2}$ in the vitiated coflow compared to the 9\% HM3 case. This trend is further observed in Fig. 6 
which shows the $\mathrm{O}_{2}$ radial distributions. Oxygen consumption in the reaction

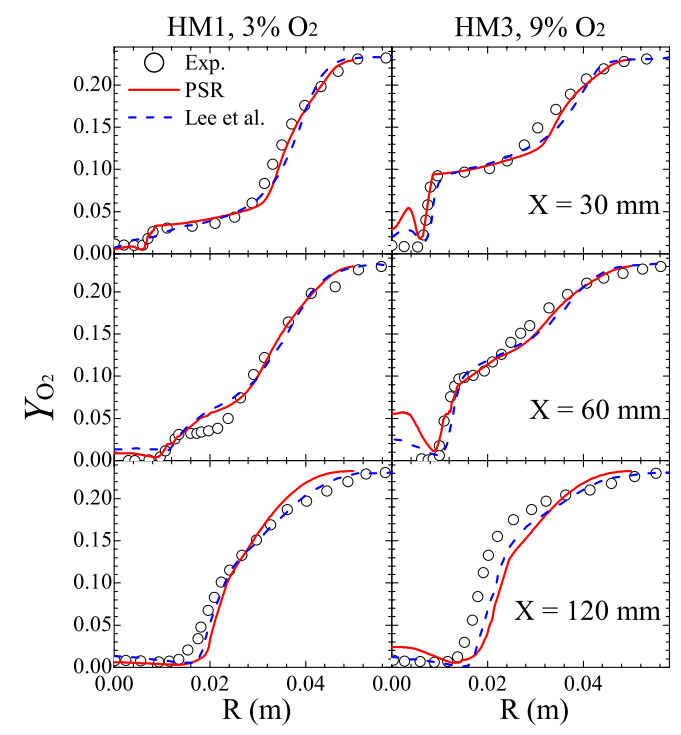

Fig. 6. Radial profiles of $\mathrm{O}_{2}$ mass fraction.

zone close to the jet centreline is well predicted by the PSR model for HM1 while a significant leakage of $\mathrm{O}_{2}$ towards the fuel rich side is seen for HM3. This is consistent with the under-estimation of temperature and $\mathrm{H}_{2} \mathrm{O}$ mass fractions observed earlier in this region. It was also suggested by Minamoto and Swaminathan $[23,24]$ using DNS data that the PSR model could under-estimate the reaction rate in the fuel rich region of MILD combustion. Nevertheless, the overall structure of this JHC configuration is well captured by the PSR model proposed in this paper concerning the mean temperature and major species mass fractions at different axial locations in the burner. The PSR model also predicts the dependence of these quantities on the oxygen-dilution level between HM1 and HM3.

The radial distribution of a minor species, $\mathrm{CO}$, is presented in Fig. 7. It is noted that there is a local maxima appearing between radial location 0.2 to $0.4 \mathrm{~m}$ 
in the experimental data at the upstream locations, which is possibly a result of the cooling and extinction near the outer wall of the secondary burner as argued by Dally et al. [14]. This local maxima does not appear in the present simulations because the presence of $\mathrm{CO}$ and other minor species in the hot coflow inlet are not taken into account and the species as reported in [14] (see Table 1) are used in the simulations. However, in the PDF calculation of Lee et al. [19], these minor species injected at the inlet were included using the profiles measured at an axial position of $X=4 \mathrm{~mm}$. Nonetheless, it is believed that this small level of CO concentration should only have minor effects on the reaction zone [14].

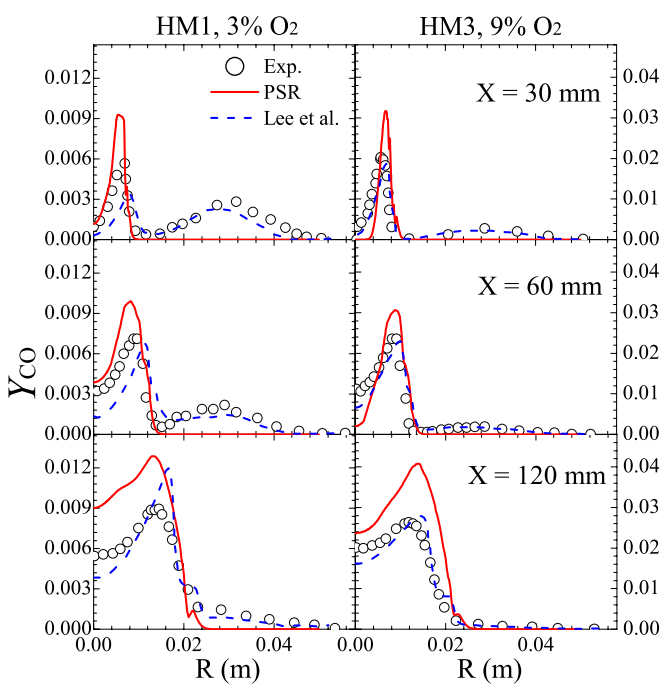

Fig. 7. Radial profiles of $\mathrm{CO}$ mass fraction.

It can be seen in Fig. 7 that the main trend and radial location of the peak are reasonably captured by the PSR model. However, a consistent over-estimation of 20-30\% is observed for the peak CO formation in both flames compared to the values measured in [14]. The multi-environment PDF model shows good predictions for flame HM3, whereas it under-estimates the CO mass fraction in the upstream 
and over-estimates in the downstream. This is expected for minor species since the transported PDF approach directly includes the multiple chemical time scales for different species and the PSR model is designated to capture the major chemical time scale in MILD combustion. Christo and Dally [15] pointed out a special trend under MILD condition where the CO formation is much more sensitive to the oxygen dilution level between HM1 and HM3 compared to $\mathrm{CO}_{2}$, which was not reproduced using the EDC model. It can been seen through the axis scales in Figs. 4 and 7 that this trend is well captured by both the PSR and PDF models using GRI-mechanisms.

\section{Conclusions}

In the present work, a modelling approach for MILD combustion has been proposed using the Perfectly Stirred Reactor. This approach is applied to simulate the Jet in Hot Coflow experimental set-up of Dally et al. [14]. The model predictions are found to be comparable to the simulation results obtain by Lee $e t$ al. [19] using a transported PDF method, showing good agreement with experimental measurements despite the simplicity of this model. The computed profiles

of mean temperature and major species mass fractions yield good comparisons with the experiments for two oxygen dilution levels. Over-prediction is observed for the formation of $\mathrm{CO}$, however, the overall trend and peak values are still captured reasonably well. This suggests that the PSR model is a promising choice for MILD combustion modelling as proposed in [23, 24, 37]. Future studies will be directed towards extending the current framework to include $\mathrm{NO}_{\mathrm{x}}$ emissions and air entrainment effects. 


\section{Acknowledgements}

ZC acknowledges the financial support of Cambridge Overseas Trusts and China Scholarship Council. SR and NS acknowledge the support of European Union funding through the project AMEL. NAKD acknowledges the financial support of the Qualcomm European Research Studentship Fund in Technology. VMR and WLR acknowledge the support from Center Competitive Fund from KAUST.

[1] J.A. Wünning, J.G. Wünning, Prog. Energy Combust. Sci. 23 (1997) 81-94.

[2] A. Cavaliere, M. de Joannon, Prog. Energy Combust. Sci. 30 (2004) 329_ 366.

[3] M. de Joannon, A. Cavaliere, T. Faravelli, E. Ranzi, P. Sabia, A. Tregrossi, Proc. Combust. Inst. 30 (2005) 2605-2612.

[4] M. de Joannon, A. Matarazzo, P. Sabia, A. Cavaliere, Proc. Combust. Inst. 31 (2007) 3409-3416.

[5] G.G. Szego, B.B. Dally, G.J. Nathan, Combust. Flame 154 (2008) 281-295.

[6] Y. Minamoto, N. Swaminathan, Combust. Flame 161 (2014) 1063-1075.

[7] Y. Minamoto, N. Swaminathan, R.S. Cant, T. Leung, Combust. Flame 161 (2014) 2801-2814.

[8] M. Katsuki, T. Hasegawa, Proc. Combust. Inst. 27 (1998) 3135-3146.

[9] S. Kumar, P.J. Paul, H.S. Mukunda, Proc. Combust. Inst. 29 (2002) 11311137. 
[10] V.M. Reddy, D. Sawant, D. Trivedi, S. Kumar, Proc. Combust. Inst. 34 (2013) 3319-3326.

[11] V.M. Reddy, A. Katoch, W.L. Roberts, S. Kumar, Proc. Combust. Inst. 35 (2015) 3581-3589.

[12] A. Parente, M.R. Malik, F. Contino, A. Cuoci, B.B. Dally, Fuel 163 (2016) $98-111$.

[13] V.K Arghode, A.K. Gupta, K.M. Bryden, Appl. Energy 92 (2012) 822-830.

[14] B.B. Dally, A.N. Karpetis, R.S. Barlow, Proc. Combust. Inst. 29 (2002) $1147-1154$.

[15] F.C. Christo, B.B. Dally, Combust. Flame 142 (2005) 117-129.

[16] J. Aminian, C. Galletti, S. Shahhosseini, L. Tognotti, Flow, Turbul. Combust. 88 (2012) 597-623.

[17] M. Ihme, J. Zhang, G. He, B.B. Dally, Flow Turbulence Combust. 89 (2012) 449-464.

[18] S.H. Kim, K.Y. Huh, B.B. Dally, Proc. Combust. Inst. 30 (2005) 751-757.

[19] J. Lee, S. Jeon, Y. Kim, Combust. Flame 162 (2015) 1464-1476.

[20] A. De, A. Dongre, Flow, Turbul. Combust. 94 (2015) 439-478.

[21] T. Plessing, N. Peters, J.G. Wünning, Proc. Combust. Inst. 27 (1998) 31973204.

[22] C. Duwig, B. Li, Z.S. Li, M. Alden, Combust. Flame 159 (2012). 
[23] Y. Minamoto, N. Swaminathan, Int. J. Adv. Eng. Sci. Appl. Math. 6 (2014) $65-75$.

[24] Y. Minamoto, N. Swaminathan, Proc. Combust. Inst. 35 (2015) 3529-3536.

[25] S. Ruan, N. Swaminathan, O.R. Darbyshire, Combust. Theory Model. 18 (2014) 295-329.

[26] Z. Chen, S. Ruan, N. Swaminathan, Combust. Flame 162 (2015) 703-716.

[27] B.B. Dally, D.F. Fletcher, A.R. Masri, Combust. Theory Model. 2 (1998) 193-219.

[28] R.W. Bilger, S.H. Stårner, R.J. Kee, Combust. Flame 80 (1990) 135-149.

[29] H. Kolla, J.W. Rogerson, N. Chakraborty, N. Swaminathan, Combust. Sci. Technol. 181 (2009) 518-535.

[30] O.R. Darbyshire, N. Swaminathan, Combust. Sci. Technol. 184 (2012) 2036-2067.

[31] O.R. Darbyshire, N. Swaminathan, S. Hochgreb, Combust. Sci. Technol. 182 (2010) 1141-1170.

[32] I. Ahmed, N. Swaminathan, Combust. Sci. Technol. 185 (2013) 1509-1540.

[33] I. Ahmed, N. Swaminathan, J. Hydrogen Energy 39 (2014) 9562-9572.

[34] S. Ruan, N. Swaminathan, M. Isono, T. Saitoh, K Saitoh, J. Propulsion Power 31 (2015) 861-871.

[35] CHEMKIN, CHEMKIN-PRO 15131, Reaction Design: San Diego, 2013. 
[36] J.A. van Oijen, L.P.H. de Goey, Combust. Sci. Technol. 161 (2000) 113-137.

[37] M. de Joannon, A. Saponaro, A. Cavaliere, Proc. Combust. Inst. 28 (2000) 1639-1646. 


\section{List of Figures}

Fig. 1: Reaction rate of progress variable in the Perfectly Stirred Reactor. (a) Temporal variation for five typical mixture fractions. (b) Distribution in the mixture fraction and progress variable spaces.

Fig. 2: Schematic of experimental geometry and computational domain. Temperature field for HM3 is shown for the region of interest and the horizontal lines denote measurement locations. The stoichiometric mixture fraction is shown as the dashed line.

Fig. 3: Radial profiles of mean temperature.

Fig. 4: Radial profiles of $\mathrm{CO}_{2}$ mass fraction.

Fig. 5: Radial profiles of $\mathrm{H}_{2} \mathrm{O}$ mass fraction.

Fig. 6: Radial profiles of $\mathrm{O}_{2}$ mass fraction.

Fig. 7: Radial profiles of CO mass fraction. 